\title{
Superconducting Zirconium Polyhydrides at Moderate Pressures
}

\author{
Hui Xie ${ }^{1, \#, ~ W e n t i n g ~ Z h a n g 1, \#, ~ D e f a n g ~ D u a n ~}{ }^{1,2, *}$, Xiaoli Huang ${ }^{1, *}$, Yanping Huang ${ }^{1}$, Hao Song ${ }^{1}$, \\ Xiaolei Feng ${ }^{3,4}$, Yansun Yao ${ }^{5}$, Chris J. Pickard ${ }^{2,6}$, Tian Cui ${ }^{1, *}$

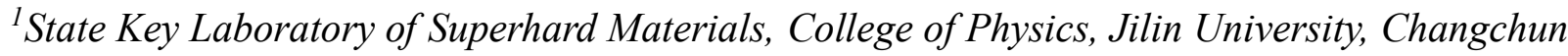 \\ 130012, China \\ ${ }^{2}$ Department of Materials Science and Metallurgy, University of Cambridge, 27 Charles Babbage \\ Road, Cambridge CB3 OFS, United Kingdom \\ ${ }^{3}$ Center for High Pressure Science and Technology Advanced Research (HPSTAR) \\ 10 Xibeiwang East Road, Beijing, 100094, China \\ ${ }^{4}$ Department of Earth Science, University of Cambridge, Downing Street, Cambridge CB2 3EQ, \\ United Kingdom \\ ${ }^{5}$ Department of Physics and Engineering Physics, University of Saskatchewan, Saskatoon, \\ Saskatchewan S7N 5E2, Canada \\ ${ }^{6}$ Advanced Institute for Materials Research, Tohoku University 2-1-1 Katahira, Aoba, Sendai, 980- \\ 8577, Japan
}

*Corresponding authors: duandf@jlu.edu.cn, huangxiaoli@jlu.edu.cn, cuitian@jlu.edu.cn

${ }^{\#}$ These authors contributed equally. 


\section{ABSTRACT}

Highly compressed hydrides have been at the forefront of the search for high- $T_{c}$ superconductivity. Especially, recent discovery of record-high $T_{c}$ 's in $\mathrm{H}_{3} \mathrm{~S}$ and $\mathrm{LaH}_{10 \pm \mathrm{x}}$ under high pressure fuels the enthusiasm finding good superconductors in similar hydride groups. Guided by first principles structure prediction, we successfully synthesized $\mathrm{ZrH}_{3}$ and $\mathrm{Zr}_{4} \mathrm{H}_{15}$ at modest pressures (30-50 GPa) in diamond anvil cells by two different reaction routes: $\mathrm{ZrH}_{2}+\mathrm{H}_{2}$ at room temperature and $\mathrm{Zr}+\mathrm{H}_{2}$ at $1500 \mathrm{~K}$ by laser heating. From the synchrotron $\mathrm{X}$-ray diffraction patterns, $\mathrm{ZrH}_{3}$ is found to have a $P m \overline{3} n$ structure corresponding to the familiar A15 structure, and $\mathrm{Zr}_{4} \mathrm{H}_{15}$ has a $I \overline{4} 3 d$ structure isostructural to $\mathrm{Th}_{4} \mathrm{H}_{15}$. Electrical resistance measurement and the dependence of $T_{c}$ on applied magnetic field of the sample showed the emergence of two superconducting transitions at 6.4 and 4.0 $\mathrm{K}$ at $40 \mathrm{GPa}$, which correspond to the two $T_{c}$ 's for $\mathrm{ZrH}_{3}$ and $\mathrm{Zr}_{4} \mathrm{H}_{15}$.

\section{Table of Contents Graphic:}
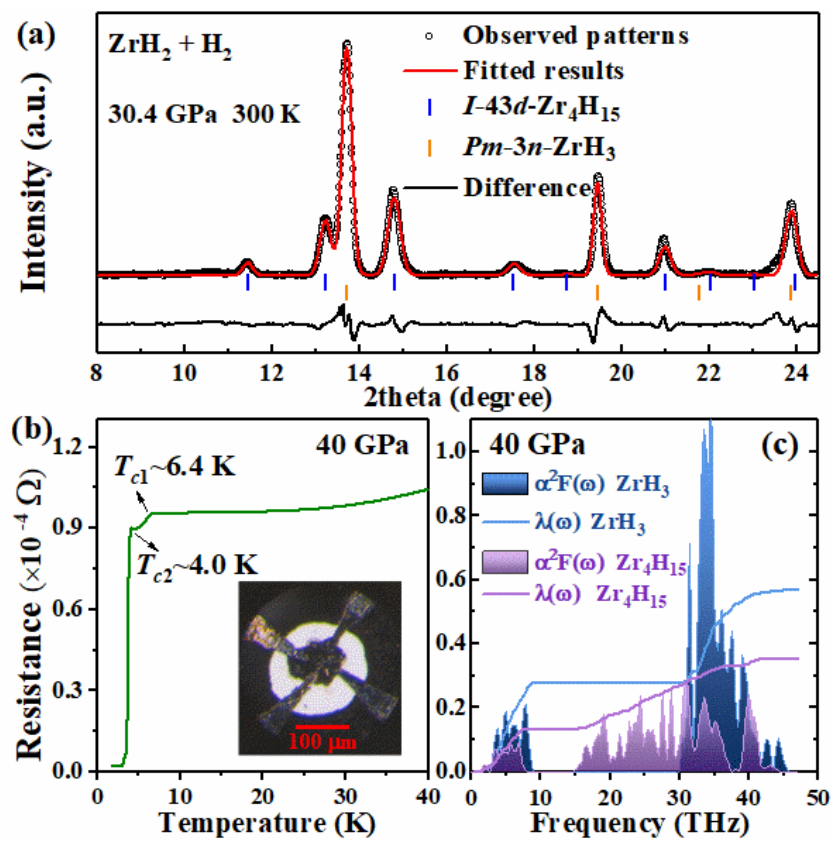
Theoretical studies suggest that highly compressed hydrogen-rich materials hold the promise as high-temperature $\left(T_{c}\right)$ superconductors ${ }^{1-3}$. Recent discovery of superconductivity above $203 \mathrm{~K}$ and 250-260 $\mathrm{K}$ in compressed $\mathrm{H}_{3} \mathrm{~S}^{4-5}$ and $\mathrm{LaH}_{10 \pm \mathrm{x}}{ }^{6-7}$ mark a milestone in superconductivity research. This discovery is groundbreaking, not only for the highest $T_{c}$ 's ever observed in all materials since the discovery of superconductivity, but also for the first time that previously unknown high- $T_{c}$ superconductors are predicted by theory ${ }^{8-10}$ and afterwards verified by experiment. Even before $\mathrm{H}_{3} \mathrm{~S}$ and $\mathrm{LaH}_{10 \pm x}$, theoretical prediction has had notable success in searching for superconducting polyhydrides. $\mathrm{SiH}_{4}$ and $\mathrm{AlH}_{3}$ were first considered due to their high contents of hydrogen among naturally-existing hydrides ${ }^{11-14}$. Experiments observed that compressed $\mathrm{SiH}_{4}$ is superconducting with maximum $T_{\mathrm{c}}$ of $17.5 \mathrm{~K}$ at $96 \mathrm{GPa}^{13}$. The experimental study confirmed the predicted structure of $\mathrm{AlH}_{3}$ but could not detect the superconductivity down to $4 \mathrm{~K}^{14}$. It was then understood that the superconductivity in $\mathrm{AlH}_{3}$ is suppressed by strong anharmonicity ${ }^{15}$. In recent years, the search for high$T_{c}$ hydrides has expanded substantially, from Group-IV to other main group hydrides ${ }^{16-17}$, and to transition metal hydrides. Many new polyhydrides have been predicted theoretically ${ }^{18-21}$, and some have already made their real-world appearance, including bulk $\mathrm{RhH}_{2}{ }^{22}, \mathrm{CoH}_{2}{ }^{23}, \mathrm{FeH}_{5}{ }^{24}$, nanocrystals $\mathrm{RhH}^{25}$, etc.

Zirconium hydrides have been studied primarily for applications in hydrogen storage and nuclear technology, but not so much as potential superconductors. For example, zirconium and its alloys are widely used as protective casings for nuclear reactor $\operatorname{cores}^{26}$. In this case, zirconium alloy operates at high temperature and forms hydride upon combination with hydrogen produced by nuclear reactions. However, we expect to find good superconducting materials in $\mathrm{Zr}-\mathrm{H}$ hydrides due to the rich structure diversity in this group. The stunning success in $\mathrm{LaH}_{10 \pm x}$ also fuels the enthusiasm finding high $T_{c}$ in similar hydride groups. Experimentally, stable $\mathrm{ZrH}$ and $\mathrm{ZrH}_{2}$, and metastable $\delta-\mathrm{ZrH}_{1.5}$ have been successfully synthesized at ambient pressure, while $\mathrm{ZrH}_{2}$ is the limit for hydrogen storage ${ }^{27-28}$. New stoichiometric $\mathrm{ZrH}_{\mathrm{n}}(n>2)$ hydrides with higher hydrogen concentration have been explored theoretically. First-principles studies up to $400 \mathrm{GPa}$ suggest $\mathrm{ZrH}_{3}$ to be stable at ambient pressure, and $\mathrm{ZrH}_{4}$ and $\mathrm{ZrH}_{6}$ to be stable above $100 \mathrm{GPa}^{29-30}$. These hydrides are calculated to be superconducting under high pressure ${ }^{30}$, which encourages the experimental realization. 


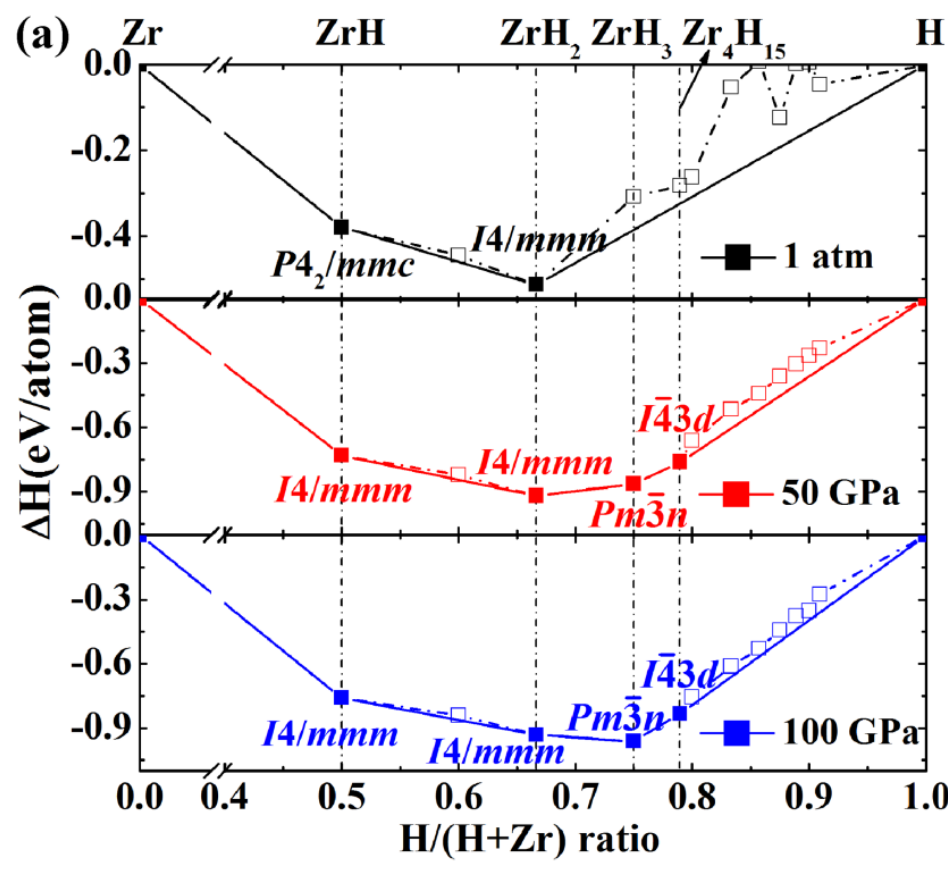

(b)

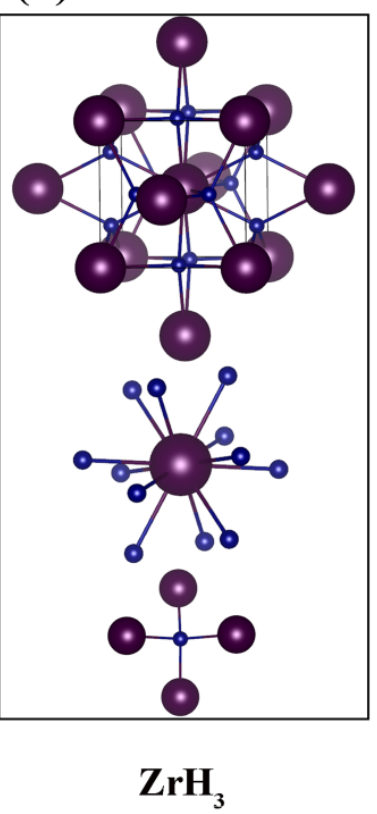

(c)

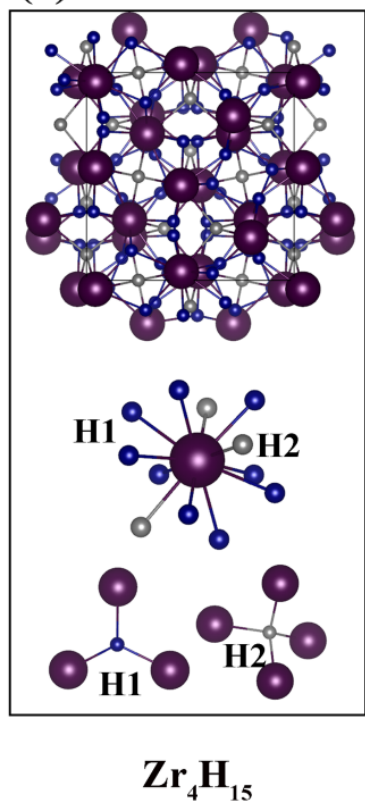

Figure 1. (a) Calculated formation enthalpies of zirconium hydrides with respect to decomposition into $\mathrm{Zr}$ and $\mathrm{H}_{2}$ under pressure, including zero-point energies. Open symbols represent unstable configurations with respect to mixing lines on the convex hull, while solid symbols on the convex hull represent stable configurations. (b) Crystal structure of $P m \overline{3} n-\mathrm{ZrH}_{3}$. (c) Conventional unit cell of $I \overline{4} 3 d$ $\mathrm{Zr}_{4} \mathrm{H}_{15}$.

Before conducting the experiment, we performed variable-composition searches for stable $\mathrm{Zr}-\mathrm{H}$ compounds at 1 atm, 50 and $100 \mathrm{GPa}$ using the CALYPSO ${ }^{31}$ and AIRSS codes ${ }^{32}$. We compared our findings with previous calculations ${ }^{29}$ and found a new stable compound $\mathrm{Zr}_{4} \mathrm{H}_{15}$ [see Figure $\mathrm{S} 1$ ]. The formation enthalpies $(\Delta \mathrm{H})$ of predicted $\mathrm{Zr}-\mathrm{H}$ hydrides with zero-point energy (ZPE) were calculated with respect to the two sets of starting materials, $\mathrm{Zr}+\mathrm{H}_{2}$ [Figure 1(a)] and $\mathrm{ZrH}_{2}+\mathrm{H}_{2}$ [Figure S2] at different pressures, from which global stability tie lines (convex hull) are constructed. Calculated $\Delta \mathrm{H}$ shows that at ambient pressure $\mathrm{ZrH}\left(\mathrm{P}_{2} / \mathrm{mmc}\right)$ and $\mathrm{ZrH}_{2}(\mathrm{I} 4 / \mathrm{mmm})$ are the only two stable stoichiometries, in agreement with experimental results ${ }^{27-28}$. At $50 \mathrm{GPa}, P m \overline{3} n-\mathrm{ZrH}_{3}$ and $I \overline{4} 3 d-\mathrm{Zr}_{4} \mathrm{H}_{15}$ enter the convex hull, indicating that they are likely synthesizable at this pressure. Meanwhile, the stable structure of $\mathrm{ZrH}$ changes to $I 4 / \mathrm{mmm}$. At $100 \mathrm{GPa}$, the available stoichiometries stay the same, but $\mathrm{ZrH}_{3}$ becomes the most stable stoichiometry. In this pressure range, all hydrides with the $\mathrm{H} / \mathrm{Zr}$ ratio higher than 15:4 are metastable. All obtained stable structures were examined by phonon calculations, which show no imaginary modes and therefore establish their dynamic stability [see Figure $\mathrm{S} 3$ ]. $\mathrm{ZrH}_{3}$ 
has A15 structure with $\operatorname{Pm} \overline{3} n$ symmetry [Figure 1(b)], in agreement with previous theoretical predictions $^{29-30}$. $\mathrm{Zr}_{4} \mathrm{H}_{15}$ has a cubic structure with $I \overline{4} 3 d$ symmetry [Figure 1(c)], which is isostructural to $\mathrm{Th}_{4} \mathrm{H}_{15}{ }^{33}$. In this structure, $\mathrm{Zr}$ atoms occupy the $16 c$ sites, while $\mathrm{H}$ atoms occupy $48 e$ (H1 in blue) and $12 a$ (H2 in gray) sites. As shown in Figure 1(c), each $\mathrm{Zr}$ atom is bonded to $12 \mathrm{H}$ atoms, among which 3 are at $12 a$ and 9 are at $48 e$. The $\mathrm{H}$ atoms at $48 e$ and $12 a$ are bonded to 3 and $4 \mathrm{Zr}$ atoms, respectively. To our best knowledge, this is the first time such structure being found in Group IVB hydrides.
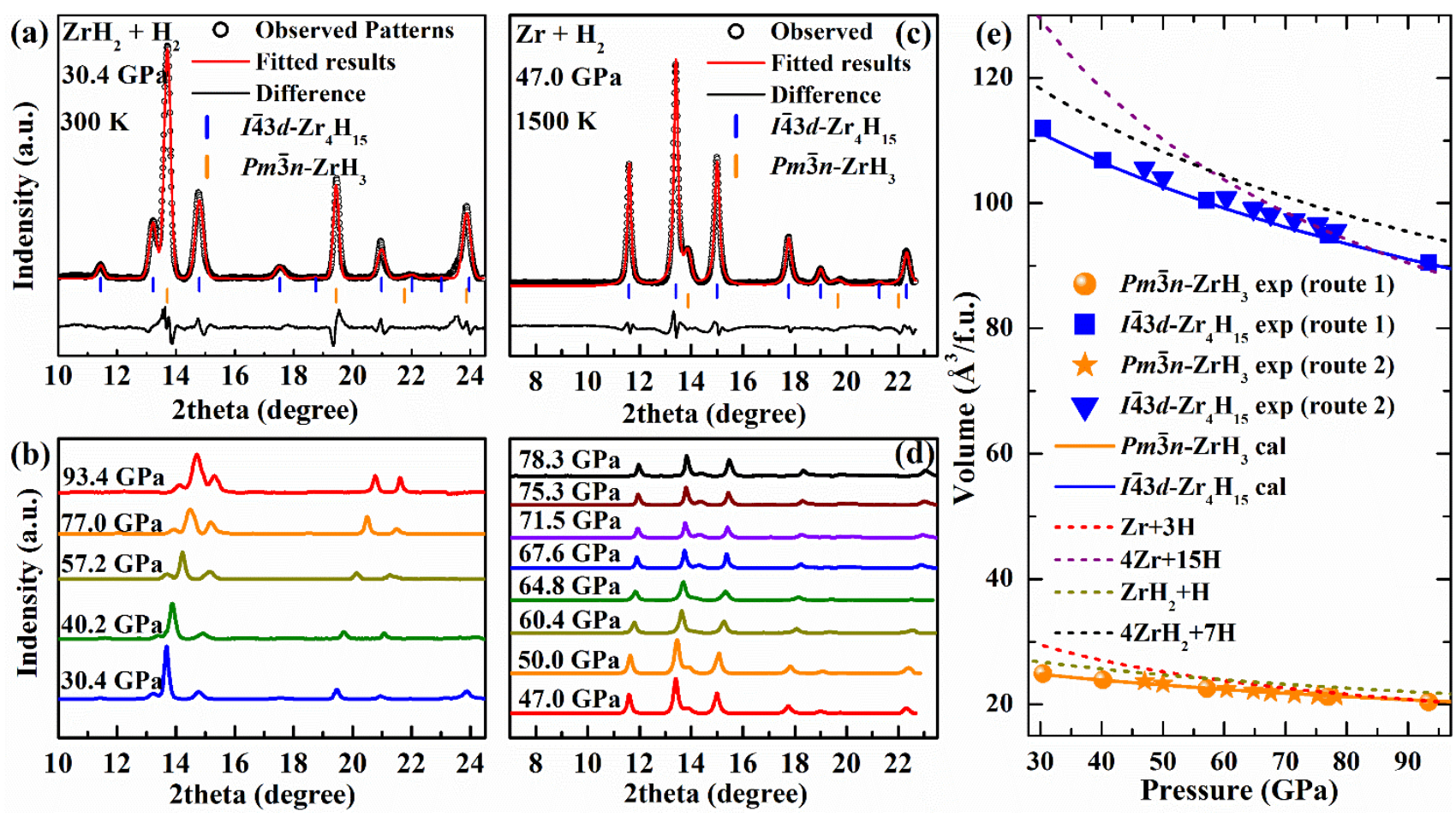

Figure 2. (a) Le Bail refinement plot of powder XRD data $(\lambda=0.6199 \AA)$ obtained in $\mathrm{ZrH}_{2}+\mathrm{H}_{2}$ reaction route at $30.4 \mathrm{GPa}$. Open circles: experimental data; red curve: model fit for the structures; blue and orange ticks: Bragg diffraction positions for $I \overline{4} 3 d-\mathrm{Zr}_{4} \mathrm{H}_{15}$ and $P m \overline{3} n-\mathrm{ZrH}_{3}$; black curve: residues. The reliability factors are $R_{p}=9.05 \%, R_{w p}=18.38 \%$. (b) Evolution of the XRD pattern of (a) with increasing pressure. (c) Refinement of the experimental XRD patterns obtained in $\mathrm{Zr}+\mathrm{H}_{2}$ reaction route at $47.0 \mathrm{GPa}$. Reliability parameters are as $R_{p}=7.44 \%, R_{w p}=12.68 \%$. (d) Evolution of the XRD pattern of (c) with increasing pressure. (e) Experimentally obtained volume per formula unit for $\mathrm{ZrH}_{3}$ and $\mathrm{Zr}_{4} \mathrm{H}_{15}$ in comparison to theoretical calculations as a function of pressure. Volumes for ideal mixtures of $\mathrm{ZrH}_{2}+\mathrm{H}, 4 \mathrm{ZrH}_{2}+7 \mathrm{H}, \mathrm{Zr}+3 \mathrm{H}$ and $4 \mathrm{Zr}+15 \mathrm{H}$ are also plotted.

With the predicted structures at hand, we performed the synthesis via two reaction routes. The first 
was to react $\mathrm{ZrH}_{2}$ with $\mathrm{H}_{2}$ at room temperature in the DAC. After the target sample was loaded into DAC, the sealed pressure was maintained at around $30.4 \mathrm{GPa}$. Figure 2(a) shows the XRD pattern collected at $30.4 \mathrm{GPa}$, in which all Bragg peaks can be indexed to either $P m \overline{3} n-\mathrm{ZrH}_{3}$ or $I \overline{4} 3 d-\mathrm{Zr}_{4} \mathrm{H}_{15}$ structure. The occurrence of a physical mixture of $\mathrm{ZrH}_{3}$ and $\mathrm{Zr}_{4} \mathrm{H}_{15}$ at this pressure is consistent to the energetic analysis [Figure 1(a)]. A Le Bail refinement of the diffraction pattern recorded at $30.4 \mathrm{GPa}$ yielded the lattice parameters for the two cubic unit cells, e.g., $a=3.676 \AA$ for $\mathrm{ZrH}_{3}$ and $a=7.636 \AA$ for $\mathrm{Zr}_{4} \mathrm{H}_{15}$. During pressurization to $93.4 \mathrm{GPa}$ [Figure 2 (b)], the diffraction peaks shift toward higher angles due to the reduction of lattice spacing but the overall pattern remains largely unchanged, indicating no other phases presenting in this pressure range. The second approach was to react $\mathrm{Zr}$ directly with $\mathrm{H}_{2}$ in a laser-heated DAC. Initially, $\mathrm{Zr}$ and $\mathrm{H}_{2}$ samples were loaded into the sample chamber of the DAC at lab conditions. The sample was then compressed to $47.0 \mathrm{GPa}$ and laser-heated to $1500 \mathrm{~K}$, from which in situ synchrotron XRD pattern was measured. As shown in Figure 2(c), the refined results show that all the observed peaks can be well indexed to $P m-3 n-\mathrm{ZrH}_{3}$ and $I-43 d-\mathrm{Zr}_{4} \mathrm{H}_{15}$ structures. Thus, the reaction products are the same as in the first reaction pathway, but the ratio of the two components seems different, as seen from the relative changes in the peak intensities. Upon further compression up to $78.3 \mathrm{GPa}$, no further transformation to any other phases was observed [Figure 2(d)].

Experimentally obtained volumes per formula unit (f. u.) for $\mathrm{ZrH}_{3}$ and $\mathrm{Zr}_{4} \mathrm{H}_{15}$, in comparison to the calculated values as functions of pressure, are plotted in Figure 2(e). Clearly, experimental equation of state (EOS) for both $\mathrm{ZrH}_{3}$ and $\mathrm{Zr}_{4} \mathrm{H}_{15}$ are in very good agreement with theory. To ascertain the identified high-pressure phases, we compare the EOS's of the two compounds to the volumes of physical mixtures representative of decomposition products at the same pressure ${ }^{34-35}$. As seen in Figure 2(e), all physical mixtures have large volumes than the $\mathrm{ZrH}_{3}$ and $\mathrm{Zr}_{4} \mathrm{H}_{15}$ compounds in the pressure range of interest, suggesting that the formation of these two compounds are thermodynamically allowed. The $P-V$ data fitted with a third-order Birch-Murnaghan (BM) equation of state ${ }^{36}$ give $V_{0}=$ $31.0(5) \AA^{3}, B_{0}=97$ (7) GPa, $B_{0}{ }^{\prime}=4$ (fixed) for $\mathrm{ZrH}_{3}$ and $V_{0}=140$ (3) $\AA^{3}, B_{0}=92$ (9) $\mathrm{GPa}, B_{0}{ }^{\prime}=4$ (fixed) for $\mathrm{Zr}_{4} \mathrm{H}_{15}$. The synthesis of $\mathrm{ZrH}_{3}$ and $\mathrm{Zr}_{4} \mathrm{H}_{15}$ represents the debut of the first Group IVB hydrides with the hydrogen-to-metal ratio greater than $2^{27,37-39}$. Zirconium is in the same group in the periodic table as rhodium, but has a smaller mass than the latter. As a result, $\mathrm{ZrH}_{3}$ and $\mathrm{Zr}_{4} \mathrm{H}_{15}$ have higher gravimetric hydrogen contents of 3.2 wt.\% and 4.0 wt. \%, respectively, as compared to 1.9 wt. $\%$ for $\mathrm{RhH}_{2}{ }^{22}$. Extrapolation from the fitted unit cell volumes for $\mathrm{ZrH}_{3}$ and $\mathrm{Zr}_{4} \mathrm{H}_{15}$ at ambient pressure 
gives two high volumetric hydrogen densities of 166.6 and $180.8 \mathrm{~g} / \mathrm{L}$, larger than that of $\mathrm{RhH}_{2}(163.7$ $\mathrm{g} / \mathrm{L}$ ). Therefore, $\mathrm{ZrH}_{3}$ and $\mathrm{Zr}_{4} \mathrm{H}_{15}$ may have the potential for hydrogen storage applications if they are recoverable to ambient conditions.

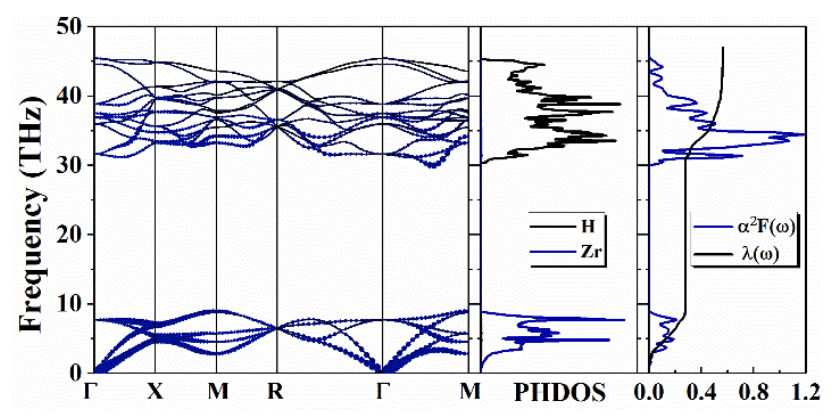

Figure 3. Phonon band structure, density of states, Eliashberg spectral function $\alpha^{2} F(\omega)$ and the EPC integral $\lambda(\omega)$ of $\mathrm{ZrH}_{3}$ at $40 \mathrm{GPa}$. Size of the circle on the phonon band structure correspond to the contribution to the EPC.

Calculated band structures and projected density of states (PDOS) of $\mathrm{ZrH}_{3}$ and $\mathrm{Zr}_{4} \mathrm{H}_{15}$ at $40 \mathrm{GPa}$ (Figure $\mathrm{S} 6$ ) show that both compounds are metallic with significant contributions of the $\mathrm{Zr}-d$ states to the DOS at the Fermi level. To investigate the phonon-mediated superconductivity, the EPC strength $\lambda$ and logarithmic average frequency $\omega_{\log }$ are calculated. The $T_{c}$ is then estimated using the AllenDynes modified McMillan equation ${ }^{40}$ with a Coulomb pseudopotential $\mu^{*}$,

$$
T_{c}=\frac{\omega_{\log }}{1.2} \exp \left[-\frac{1.04(1+\lambda)}{\lambda-\mu^{*}(1+0.62 \lambda)}\right] .
$$

The calculated $\lambda$ for $\mathrm{Zr}_{4} \mathrm{H}_{15}$ is 0.33 at $40 \mathrm{GPa}$, and the $\omega_{\log }$ is $792 \mathrm{~K}$. Using a nominal value 0.13 for $\mu^{*}$, the estimated $T_{c}$ at $40 \mathrm{GPa}$ are $0.2 \mathrm{~K}$ [see Table 1]. Likewise, the calculated $\lambda$ for $\mathrm{ZrH}_{3}$ are 0.68 , 0.61 , and 0.57 at 10,20 and $40 \mathrm{GPa}$, and the $\omega_{\log }$ are 518,571 , and $646 \mathrm{~K}$, respectively. Using $\mu^{*}=$ 0.13 the estimated $T_{c}$ for $\mathrm{ZrH}_{3}$ at 10,20 , and $40 \mathrm{GPa}$ are $12.5,9.8$, and $8.4 \mathrm{~K}$, respectively. $\mathrm{ZrH}_{3}$ is therefore predicted to have a higher $T_{c}$ than $\mathrm{Zr}_{4} \mathrm{H}_{15}$. To elucidate the mechanism, phonon dispersion curves and density of states (PHDOS), Eliashberg spectral function $\alpha^{2} F(\omega)$ and EPC integral $\lambda(\omega)$ are calculated for $\mathrm{ZrH}_{3}$ at $40 \mathrm{GPa}$ [Figure. 3]. For the calculated $\lambda$ of 0.57 , the high-frequency $\mathrm{H}$ vibrational modes contribute about $51 \%$. To identify the contributions of various phonon modes along different vibrational directions, solid circles with the radius proportional to the EPC strength are plotted on the phonon dispersion curves. Interestingly, the projected EPC along different directions are almost equal. 

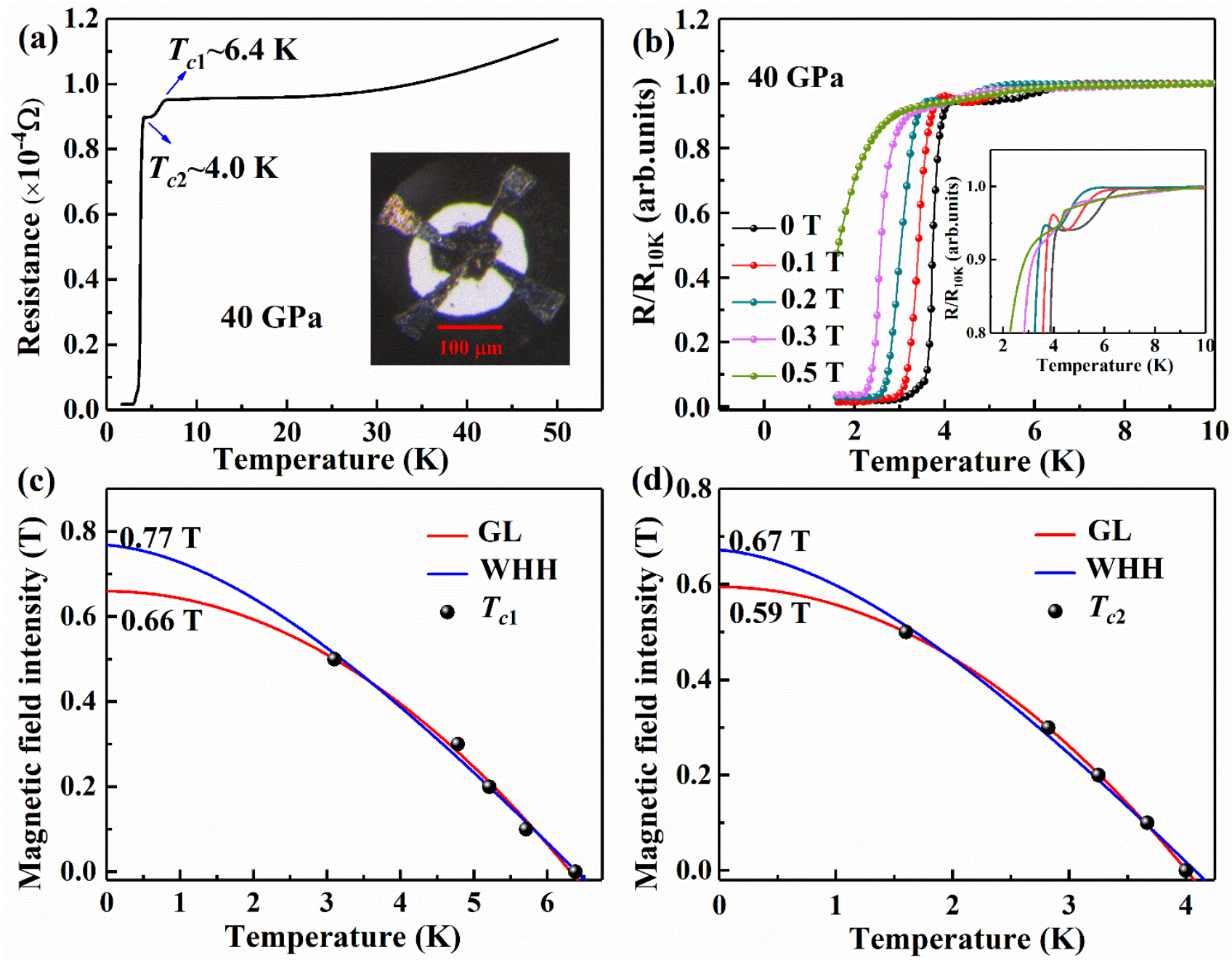

Figure 4. (a) Electrical resistance $R(\mathrm{~T})$ curve of $\mathrm{Zr}-\mathrm{H}$ sample at $40 \mathrm{GPa}$. Inset shows the photo of the sample after laser heating. (b) The superconducting transition of the sample with different applied magnetic field at $40 \mathrm{GPa}$. (c) and (d) The dependence of $T_{\mathrm{c} 1}$ and $T_{\mathrm{c} 2}$ on the external magnetic field $H_{\mathrm{c}}$. The solid lines in blue and red represent fittings by WHH and GL equation, respectively.

To investigate the predicted superconductivity in $\mathrm{ZrH}_{3}$ and $\mathrm{Zr}_{4} \mathrm{H}_{15}$, we carried out high-pressure variable-temperature electrical resistance measurements. To make the sample, we used $\mathrm{NH}_{3} \mathrm{BH}_{3}$ as the hydrogen source. The sample containing $\mathrm{ZrH}_{2}$ and $\mathrm{NH}_{3} \mathrm{BH}_{3}$ was initially compressed to $30 \mathrm{GPa}$ in the DAC and then laser-heated to $1600 \mathrm{~K}$. After the reaction, the sample was compressed to $40 \mathrm{GPa}$, at where two superconducting transitions were detected with pronounced zero resistance [Figure 4(a)]. We proposed that these correspond to two $T_{c}$ 's for the two phases, one at $6.4 \mathrm{~K}\left(T_{\mathrm{c} 1}\right)$ and the other at $4.0 \mathrm{~K}\left(T_{\mathrm{c} 2}\right)$. We first ruled out the possibility that these two superconducting transitions belong to $\mathrm{BN}$, $\mathrm{NH}_{3} \mathrm{BH}_{3}$ or $\mathrm{ZrH}_{2}$ [Figure S6]. From the XRD pattern of the prepared sample deposited with four electrodes, the sample contains both $\mathrm{ZrH}_{3}$ and $\mathrm{Zr}_{4} \mathrm{H}_{15}$ [Figure S7]. Based on our calculation [Table 1], 
we tentatively interpret that the higher $T_{c}$ is likely to be that of $\mathrm{ZrH}_{3}$, and the lower one is for $\mathrm{Zr}_{4} \mathrm{H}_{15}$. The superconducting resistance drop $R(\mathrm{~T})$ is also dependent on the applied magnetic field, further proving these two superconducting transitions. As demonstrated in Figure 4(b), the two superconducting transition temperatures $T_{\mathrm{c} 1}$ and $T_{\mathrm{c} 2}$ shift to lower temperatures upon increasing the magnetic field $\left(H_{\mathrm{c}}\right)$ to $0.5 \mathrm{~T}$. To determine the upper critical magnetic field $H_{\mathrm{c}}(0)$, the extrapolation methods combined with the Ginzburg-Landau (GL) equation ${ }^{41}$ and Werthdamer-Helfand-Hohenberg (WHH) equation ${ }^{42}$ are shown in Figures. 4(c) and 4(d), respectively.

Table 1. Calculated superconducting transition temperatures $T_{c}(\mathrm{~K}), \mathrm{EPC}$ constant $\lambda$, logarithmic average phonon frequency $\omega_{\log }(\mathrm{K})$, electronic DOS at the Fermi level $\mathrm{N}\left(\varepsilon_{\mathrm{f}}\right)$ (states/eV/f.u.), average of squared phonon frequency $\left\langle\omega^{2}\right\rangle\left(\mathrm{THz}^{2}\right)$ and average electron-phonon matrix element $\left\langle\mathrm{I}^{2}\right\rangle$ $\left(\mathrm{eV}^{3} /\right.$ states). Percentages in parentheses represent the contribution of $\mathrm{H}$ atoms to the total $\lambda$ and $\mathrm{N}\left(\varepsilon_{\mathrm{f}}\right)$.

\begin{tabular}{|c|c|c|c|c|c|c|}
\hline \multirow{2}{*}{ Hydrides (Pressure) } & $T_{c}(\mathrm{~K})$ & \multirow{2}{*}{$\lambda$} & \multirow{2}{*}{$\omega_{\log }$} & \multirow{2}{*}{$N\left(\varepsilon_{\mathrm{f}}\right)$} & \multirow{2}{*}{$<\omega^{2}>$} & \multirow{2}{*}{$<\mathrm{I}^{2}>$} \\
\hline & $\left(\mu^{*}=0.1-0.13\right)$ & & & & & \\
\hline $\mathrm{Zr}_{4} \mathrm{H}_{15}(40 \mathrm{GPa})$ & $0.2-0.8$ & 0.33 & 792 & 3.55 & I & I \\
\hline $\mathrm{ZrH}_{3}(10 \mathrm{GPa})$ & $12.5-16.6$ & 0.68 & 518 & 0.88 & l & / \\
\hline $\mathrm{ZrH}_{3}(20 \mathrm{GPa})$ & $9.8-13.8$ & 0.61 & 571 & 0.85 & I & I \\
\hline $\mathrm{ZrH}_{3}(40 \mathrm{GPa})$ & $8.4-12.4$ & $0.57(51 \%)$ & 646 & $0.81(10 \%)$ & 650.6 & 0.74 \\
\hline $\mathrm{H}_{3} \mathrm{~S}(200 \mathrm{GPa})$ & $211.6-230.2$ & $1.88(>80 \%)$ & 1266 & $0.6(43 \%)$ & 962.2 & 1.81 \\
\hline
\end{tabular}

It is generally accepted that anharmonicity can have noticeable influence on phonon-mediated superconductivity $^{15,43}$, especially in structures with softened phonon modes. For example, in $P m \overline{3} n$ $\mathrm{AlH}_{3}$, anharmonicity can suppress the EPC and reduces the $T_{\mathrm{c}}$ from $24 \mathrm{~K}$ to $2 \mathrm{~K}$, giving the explanation for the discrepancy between experimental and theoretical results ${ }^{15}$. Although $\mathrm{ZrH}_{3}$ has the same structure as $\mathrm{AlH}_{3}$, its flat phonon spectrum implies that anharmonic effects on EPC is small. For $\mathrm{ZrH}_{3}$, experimentally determined $T_{\mathrm{c}}$ of $6.4 \mathrm{~K}$ is slight lower than the theoretical value of $\sim 8.4 \mathrm{~K}$. A possible reason for this discrepancy is that the actual Coulomb pseudopotential $\mu^{*}$ in $\mathrm{ZrH}_{3}$ is greater than 1.3, approximately equal to 1.5 . As pointed out previously ${ }^{44}$, the effects of Coulomb repulsion can be very large in low-density metals and it would be substantially underestimated if the nominal value is used in the McMillan equation, and this may be the case for zirconium hydrides. Also of interest is the 
puzzle why $\mathrm{ZrH}_{3}$ has same stoichiometry and similar structure as $\mathrm{H}_{3} \mathrm{~S}$ but does not have a $T_{c}$ as nearly close to the latter. The $\mathrm{H}_{3} \mathrm{~S}$ under the pressure of $153 \mathrm{GPa}$ shows both a Meissner effect and zero electrical resistivity with a high $T_{c}$ of $203 \mathrm{~K}$. To probe the relative superconductivity of $\mathrm{ZrH}_{3}$ and $\mathrm{H}_{3} \mathrm{~S}$, we compared their $T_{c}$ 's and associated parameters in Table 1 . We note that the $\lambda$ and $\omega_{\log }$ in $\mathrm{ZrH}_{3}$ are significantly smaller than those for $\mathrm{H}_{3} \mathrm{~S}$, which explains the fairly low $T_{c}$ in $\mathrm{ZrH}_{3}$. According to the Eq. 1 , the $T_{c}$ is linearly proportional to $\omega_{\log }$ Numerically, $\omega_{\log }$ of $\mathrm{H}_{3} \mathrm{~S}$ is about twice that of $\mathrm{ZrH}_{3}$, but $T_{c}$ of $\mathrm{H}_{3} \mathrm{~S}$ is 19-25 times larger than $\mathrm{ZrH}_{3}$, indicating that the EPC strength $\lambda$ dominates the estimated $T_{c}$ [Table I]. As mentioned above, the electron-phonon interaction of hydrogen contributes $51 \%$ to the total $\lambda$ in $\mathrm{ZrH}_{3}$, much lower than that over $80 \%$ in $\mathrm{H}_{3} \mathrm{~S}$. To further explore the electron-phonon coupling, we note that ${ }^{45}$ :

$$
\lambda=\frac{N\left(\varepsilon_{f}\right)\left\langle I^{2}\right\rangle}{M\left\langle\omega^{2}\right\rangle},\left\langle\omega^{2}\right\rangle=\frac{2}{\lambda} \int \alpha^{2} F(\omega) \omega d \omega
$$

where $\left\langle I^{2}\right\rangle$ is the average over the Fermi surface of the electron-phonon matrix element and M is the atomic mass. Given that $\mathrm{M}$ is a constant, we present the other three parameters in Table I. As is shown, although $\mathrm{ZrH}_{3}$ has a larger DOS at the Fermi level $\mathrm{N}\left(\varepsilon_{\mathrm{f}}\right)$ than $\mathrm{H}_{3} \mathrm{~S}$, the $\mathrm{H}$ states contribute much less, which is not favorable to EPC. In addition, a larger mass for metal element and smaller electronphonon matrix element also result in a weaker $\lambda$ of $\mathrm{ZrH}_{3}$, giving a much lower $T_{c}$ than that of $\mathrm{H}_{3} \mathrm{~S}$.

In summary, a mixture phase of $\mathrm{ZrH}_{3}$ and $\mathrm{Zr}_{4} \mathrm{H}_{15}$ was obtained by direct reaction of $\mathrm{Zr}+\mathrm{H}_{2}$ at 47.0 $\mathrm{GPa}$, and by reacting $\mathrm{ZrH}_{2}+\mathrm{H}_{2}$ at $30.4 \mathrm{GPa}$. Upon increasing the pressure, the two phases persist to 93.4 GPa without any phase transition or decomposition. $\mathrm{ZrH}_{3}$ and $\mathrm{Zr}_{4} \mathrm{H}_{15}$ are isostructural to A15 and $\mathrm{Th}_{4} \mathrm{H}_{15}$ structures, respectively. This is the first time that stoichiometry like $\mathrm{ZrH}_{3}$ and $\mathrm{Zr}_{4} \mathrm{H}_{15}$ is realized for Group IVB hydrides. Theoretical prediction, electrical resistance and external magnetic experiments reveal that both $\mathrm{ZrH}_{3}$ and $\mathrm{Zr}_{4} \mathrm{H}_{15}$ are conventional superconducting materials, with the measured superconducting transition temperatures of $6.4 \mathrm{~K}$ and $4.0 \mathrm{~K}$ at $40 \mathrm{GPa}$, respectively. Our results provide a reference for the synthesis of new hydrogen-rich materials under high pressures and the exploration of metal hydrides. Polyhydrides of zirconium with higher hydrogen content may exist at even higher pressures suggesting the need for further experiments to explore these systems.

\section{AUTHOR INFORMATION}

Author Contributions: D. D. and T. C. designed research; H. X. D. D and C. P performed theoretical calculation; W. Z. X. H and Y. H. performed the experiment; H. X., D. D., X. H., Y. H., H. S., Y. Y., C. 
P. and T. C. analyzed data; H. X., X. F., D. D., X. H., Y. Y., C. P. and T. C. wrote the paper.

\section{Corresponding Authors:}

*E-mail: duandf@jlu.edu.cn (D. D.)

*E-mail: huangxiaoli@jlu.edu.cn (X. H.)

*E-mail: cuitian@jlu.edu.cn (T. C.)

\section{Notes}

The authors declare no conflict of interest.

\section{ACKNOWLEDGEMENTS}

This work was supported by the National Key R\&D Program of China (No. 2018YFA0305900), National Natural Science Foundation of China (Nos. 51632002, 11674122, 51572108, 11504127), Program for Changjiang Scholars and Innovative Research Team in University (No. IRT_15R23), the 111 Project (No. B12011), and the Natural Sciences and Engineering Research Council of Canada (NSERC). CJP acknowledges financial support from the Engineering and Physical Sciences Research Council [Grant EP/P022596/1] and a Royal Society Wolfson Research Merit award. Parts of calculations were performed in the High Performance Computing Center (HPCC) of Jilin University and TianHe-1(A) at the National Supercomputer Center in Tianjin. 


\section{REFERENCES}

(1) Duan, D.; Liu, Y.; Ma, Y.; Shao, Z.; Liu, B.; Cui, T. Structure and superconductivity of hydrides at high pressures. Natl. Sci. Rev. 2016, 4, 121-135.

(2) Gor'kov, L. P.; Kresin, V. Z. Colloquium: High pressure and road to room temperature superconductivity. Rev. Mod. Phys. 2018, 90, 011001.

(3) Zurek, E.; Bi, T. High-temperature superconductivity in alkaline and rare earth polyhydrides at high pressure: A theoretical perspective. J. Chem. Phys. 2019, 150, 050901.

(4) Drozdov, A. P.; Eremets, M. I.; Troyan, I. A.; Ksenofontov, V.; Shylin, S. I. Conventional superconductivity at 203 kelvin at high pressures in the sulfur hydride system. Nature 2015, 525, 73.

(5) Einaga, M.; Sakata, M.; Ishikawa, T.; Shimizu, K.; Eremets, M. I.; Drozdov, A. P.; Troyan, I. A.; Hirao, N.; Ohishi, Y. Crystal structure of the superconducting phase of sulfur hydride. Nat. Phys. 2016, 12, 835.

(6) Drozdov, A. P.; Kong, P. P.; Minkov, V. S.; Besedin, S. P.; Kuzovnikov, M. A.; Mozaffari, S.; Balicas, L.; Balakirev, F. F.; Graf, D. E.; Prakapenka, V. B., et al. Superconductivity at $250 \mathrm{~K}$ in lanthanum hydride under high pressures. Nature 2019, 569, 528-531.

(7) Somayazulu, M.; Ahart, M.; Mishra, A. K.; Geballe, Z. M.; Baldini, M.; Meng, Y.; Struzhkin, V. V.; Hemley, R. J. Evidence for Superconductivity above $260 \mathrm{~K}$ in Lanthanum Superhydride at Megabar Pressures. Phys. Rev. Lett. 2019, 122, 027001.

(8) Duan, D.; Liu, Y.; Tian, F.; Li, D.; Huang, X.; Zhao, Z.; Yu, H.; Liu, B.; Tian, W.; Cui, T. Pressure-induced metallization of dense $\left(\mathrm{H}_{2} \mathrm{~S}\right)_{2} \mathrm{H}_{2}$ with high-Tc superconductivity. Sci. Rep. 2014, 4, 6968.

(9) Liu, H.; Naumov, I. I.; Hoffmann, R.; Ashcroft, N. W.; Hemley, R. J. Potential high- $T_{c}$ superconducting lanthanum and yttrium hydrides at high pressure. Proc. Natl. Acad. Sci. U. S. A. 2017, 114, 6990-6995.

(10) Peng, F.; Sun, Y.; Pickard, C. J.; Needs, R. J.; Wu, Q.; Ma, Y. Hydrogen Clathrate Structures in Rare Earth Hydrides at High Pressures: Possible Route to Room-Temperature Superconductivity. Phys. Rev. Lett. 2017, 119, 107001.

(11) Pickard, C. J.; Needs, R. J. High-pressure phases of silane. Phys. Rev. Lett. 2006, 97, 045504.

(12) Pickard, C. J.; Needs, R. J. Metallization of aluminum hydride at high pressures: A first-principles study. Phys. Rev. B 2007, 76, 144114.

(13) Eremets, M. I.; Trojan, I. A.; Medvedev, S. A.; Tse, J. S.; Yao, Y. Superconductivity in Hydrogen Dominant Materials: Silane. Science 2008, 319, 1506.

(14) Goncharenko, I.; Eremets, M. I.; Hanfland, M.; Tse, J. S.; Amboage, M.; Yao, Y.; Trojan, I. A. Pressureinduced hydrogen-dominant metallic state in aluminum hydride. Phys. Rev. Lett. 2008, 100, 045504.

(15) Rousseau, B.; Bergara, A. Giant anharmonicity suppresses superconductivity in $\mathrm{AlH}_{3}$ under pressure. Phys. Rev. B 2010, 82, 104504.

(16) Shamp, A.; Zurek, E. Superconducting High-Pressure Phases Composed of Hydrogen and Iodine. J. Phys. Chem. Lett. 2015, 6, 4067-4072.

(17) Shamp, A.; Terpstra, T.; Bi, T.; Falls, Z.; Avery, P.; Zurek, E. Decomposition Products of Phosphine Under Pressure: PH2 Stable and Superconducting? J. Am. Chem. Soc. 2016, 138, 1884-1892.

(18) Wang, L.; Duan, D.; Yu, H.; Xie, H.; Huang, X.; Ma, Y.; Tian, F.; Li, D.; Liu, B.; Cui, T. High-Pressure Formation of Cobalt Polyhydrides: A First-Principle Study. lnorg. Chem. 2018, 57, 181-186.

(19) Gao, G.; Wang, H.; Zhu, L.; Ma, Y. Pressure-Induced Formation of Noble Metal Hydrides. J. Phys. Chem. C 2012, 116, 1995-2000.

(20) Kvashnin, A. G.; Semenok, D. V.; Kruglov, I. A.; Wrona, I. A.; Oganov, A. R. High-Temperature Superconductivity in a Th-H System under Pressure Conditions. ACS Appl. Mater. Inter. 2018, 10, 43809-43816. (21) Semenok, D. V.; Kvashnin, A. G.; Kruglov, I. A.; Oganov, A. R. Actinium Hydrides $\mathrm{AcH}_{10}, \mathrm{AcH}_{12}$, and 
$\mathrm{AcH}_{16}$ as High-Temperature Conventional Superconductors. J. Phys. Chem. Lett. 2018, 9, 1920-1926.

(22) Li, B.; Ding, Y.; Kim, D. Y.; Ahuja, R.; Zou, G.; Mao, H. K. Rhodium dihydride $\left(\mathrm{RhH}_{2}\right)$ with high volumetric hydrogen density. Proc. Natl. Acad. Sci. U. S. A. 2011, 108, 18618-21.

(23) Wang, M.; Binns, J.; Donnelly, M.-E.; Peña-Alvarez, M.; Dalladay-Simpson, P.; Howie, R. T. High pressure synthesis and stability of cobalt hydrides. J. Chem. Phys. 2018, 148, 144310.

(24) Pépin, C. M.; Geneste, G.; Dewaele, A.; Mezouar, M.; Loubeyre, P. Synthesis of FeH ${ }_{5}$ : A layered structure with atomic hydrogen slabs. Science 2017, 357, 382.

(25) Liu, C.; Jiang, S.; Sui, Y.; Chen, Y.; Xiao, G.; Chen, X.-J.; Shu, H.; Duan, D.; Li, X.; Liu, H., et al. Effect of the Inherent Structure of Rh Nanocrystals on the Hydriding Behavior under Pressure. J. Phys. Chem. Lett. 2019, 10, 774-779.

(26) Singh, R. N.; Bind, A. K.; Srinivasan, N. S.; Ståhle, P. Influence of hydrogen content on fracture toughness of CWSR Zr-2.5Nb pressure tube alloy. J. Nucl. Mater. 2013, 432, 87-93.

(27) Zuzek, E.; Abriata, J. P.; San-Martin, A.; Manchester, F. D. The H-Zr (hydrogen-zirconium) system. Bull. Alloy Phase Diagrams 1990, 11, 385-395.

(28) Root, J. H.; Small, W. M.; Khatamian, D.; Woo, O. T. Kinetics of the $\delta$ to $\gamma$ zirconium hydride transformation in Zr-2.5Nb. Acta Mater. 2003, 51, 2041-2053.

(29) Li, X. F.; Hu, Z. Y.; Huang, B. Phase diagram and superconductivity of compressed zirconium hydrides. Phys. Chem. Chem. Phys. 2017, 19, 3538-3543.

(30) Abe, K. High-pressure properties of dense metallic zirconium hydrides studied by ab initio calculations. Phys. Rev. B 2018, 98, 134103.

(31) Wang, Y.; Lv, J.; Zhu, L.; Ma, Y. Crystal structure prediction via particle-swarm optimization. Phys. Rev. B 2010, 82, 094116.

(32) Pickard, C. J.; Needs, R. J. Ab initiorandom structure searching. J. Phys.: Condens. Matter 2011, 23, 053201.

(33) Zachariasen, W. Crystal chemical studies of the $5 \mathrm{f}$-series of elements. XIX. The crystal structure of the higher thorium hydride, $\mathrm{Th}_{4} \mathrm{H}_{15}$. Acta Crystallogr. 1953, 6, 393-395.

(34) Loubeyre, P.; LeToullec, R.; Hausermann, D.; Hanfland, M.; Hemley, R. J.; Mao, H. K.; Finger, L. W. Xray diffraction and equation of state of hydrogen at megabar pressures. Nature 1996, 383, 702-704.

(35) Stavrou, E.; Yang, L. H.; Söderlind, P.; Aberg, D.; Radousky, H. B.; Armstrong, M. R.; Belof, J. L.; Kunz, M.; Greenberg, E.; Prakapenka, V. B., et al. Anharmonicity-induced first-order isostructural phase transition of zirconium under pressure. Phys. Rev. B 2018, 98, 220101.

(36) Birch, F. The Effect of Pressure Upon the Elastic Parameters of Isotropic Solids, According to Murnaghan's Theory of Finite Strain. J. Appl. Phys 1938, 9, 279-288.

(37) Sidhu, S. S.; McGuire, J. C. An X - Ray Diffraction Study of the Hafnium - Hydrogen System. J. Appl. Phys 1952, 23, 1257-1261.

(38) Sidhu, S. S. The effect on metal-metal bonds of increased concentration of hydrogen in hafnium dihydride. Acta Crystallogr. 1954, 7, 447-449.

(39) Kalita, P. E.; Sinogeikin, S. V.; Lipinska-Kalita, K.; Hartmann, T.; Ke, X.; Chen, C.; Cornelius, A. Equation of state of $\mathrm{TiH}_{2}$ up to $90 \mathrm{GPa}$ : A synchrotron x-ray diffraction study and ab initio calculations. J. Appl. Phys 2010, $108,043511$.

(40) Allen, P. B.; Dynes, R. C. Transition temperature of strong-coupled superconductors reanalyzed. Phys. Rev. B 1975, 12, 905-922.

(41) Woollam, J. A.; Somoano, R. B.; O'Connor, P. Positive Curvature of the $H_{c 2}$-versus- $T_{c}$ Boundaries in Layered Superconductors. Phys. Rev. Lett. 1974, 32, 712-714. 
(42) Baumgartner, T.; Eisterer, M.; Weber, H. W.; Flükiger, R.; Scheuerlein, C.; Bottura, L. Effects of neutron irradiation on pinning force scaling in state-of-the-art $\mathrm{Nb}_{3} \mathrm{Sn}$ wires. Supercond. Sci. Technol. 2014, 27, 015005. (43) Errea, I.; Calandra, M.; Pickard, C. J.; Nelson, J.; Needs, R. J.; Li, Y.; Liu, H.; Zhang, Y.; Ma, Y.; Mauri, F. High-Pressure Hydrogen Sulfide from First Principles: A Strongly Anharmonic Phonon-Mediated Superconductor. Phys. Rev. Lett. 2015, 114, 157004.

(44) Richardson, C. F.; Ashcroft, N. W. High Temperature Superconductivity in Metallic Hydrogen: ElectronElectron Enhancements. Phys. Rev. Lett. 1997, 78, 118-121.

(45) McMillan, W. L. Transition Temperature of Strong-Coupled Superconductors. Phys. Rev. 1968, 167, 331344. 